\title{
Augmented Reality during Angiography: Integration of a Virtual Mirror for Improved 2D/3D Visualization
}

\author{
Jian Wang, Pascal Fallavollita*, Lejing Wang, Matthias Kreiser, and Nassir Navab \\ Chair for Computer Aided Medical Procedures, Fakultät für Informatik, Technische Universität München
}

\begin{abstract}
Visualizing 2D and 3D anatomical information simultaneously within an X-ray image is challenging. Multiple monitors are required in the operating room to enable visualization of anatomical data by the surgeon. Consequently, this results in an interruption during the operation for proper assessment of information. In this paper, we introduce an interactive visualization of the $3 \mathrm{D}$ data from new perspectives including visualizing a virtual mirror from the same viewpoint as the X-ray source. The main contribution is our development of a complete angiographic visualization system that displays simultaneous $2 \mathrm{D}$ $\mathrm{X}$-ray and 3D anatomical information in a common monitor for the surgeon in the operating room. No previous works have conceived the integration of the virtual mirror into the projection geometry of a C-arm fluoroscope. In total, 24 participants were asked to assess the benefits of the angiographic virtual mirror with different colour-depth encodings. The results of our feasibility study show a clear improvement when deciphering the true positions of aneurysms in X-ray. Lastly, color depth encoding improves correspondence between the $3 \mathrm{D}$ vasculature displayed in the virtual mirror to their projection images in X-ray.
\end{abstract}

Keywords: Augmented Reality, Angiography, Virtual Mirror, Xray, Visualization, 2D/3D Registration.

Index Terms: H.5.1 [Multimedia Information Systems]: artificial, augmented, and virtual realities; H.5.2 [Information Interfaces and presentation]: Interaction styles-Evaluation/methodology; I.2.1. [Applications and Expert Systems] — medicine and science

\section{INTRODUCTION}

In current clinical practice, 3D augmented fluoroscopy involves superimposing preoperative data onto a live intraoperative X-ray. This is known as a 2D/3D overlay. In the past decade, accuracy of the $2 \mathrm{D} / 3 \mathrm{D}$ overlay has been the most regarded problem in research practice. A wide range of topics tackling this problem have been surveyed by Markelj et al. [1]. Proper interpretation and perception of the overlay is crucial to clinicians during intervention. From this point of view, few works have been published on improving the visualization of information in a single X-ray image.

In the past, volume data generated by CT or MRI could only be viewed as individual slices. This resulted in strenuous cognitive tasks for clinicians to fully appreciate the spatial layout [6]. Volume visualization has been continuously challenging in the computer graphics community. Depth perception is an important facet in volume rendering. Bruckner and Gröller introduce

* corresponding author email: fallavol@in.tum.de

IEEE International Symposium on Mixed and Augmented Reality 2012 Science and Technology Proceedings

5 - 8 November 2012, Atlanta, Georgia

978-1-4673-4661-0/12/\$31.00 @2012 IEEE

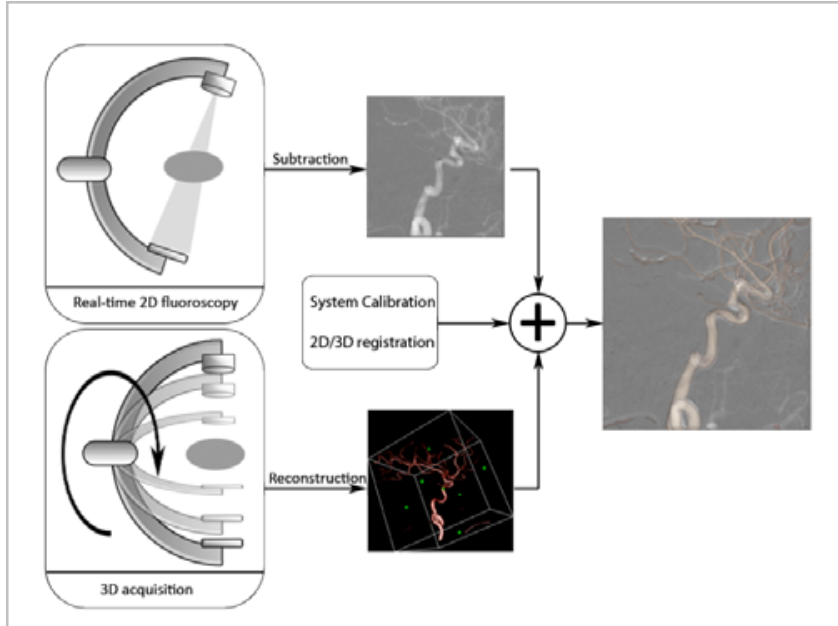

Figure 1: Example of 2D/3D overlay during angiography.

volumetric halos in the volume rendering pipeline to facilitate depth perception. The halos are known as the darkened or brightened regions surrounding the edges of specific structures. The added halos help judge the spatial relationships more accurately [2]. The order-independent volume rendering techniques, such as MIP (maximum intensity projection) and DRR (digital reconstructed radiograph) rendering, typically have poor depth perception. Mora and Ebert introduced the orderindependent volume rendering (OIVR) techniques to improve MIP and X-ray using gradient signals inside stereo images. Instead of using only the voxel data to produce the MIP or DRRs, the gradient magnitude was used in the rendering to convey additional information [3]. Kersten et al. used purely absorptive lighting model in DRR rendering to enhance the depth perception of the synthetic X-ray images [4]. Lastly, Wieczorek et al. recently introduced an interactive X-Ray perceptual visualization (IXPV) technique to improve 3D perception in X-Ray. With preoperative data, it allows the user to interactively manipulate the $\mathrm{X}$-ray image by varying depth [5]. However, none of these techniques visualize simultaneously in X-ray the overlay and the complete 3D anatomy of interest.

Today, most interventionalists and radiologists are better trained to intuitively gather information from a two dimensional $\mathrm{X}$-ray image since it remains the primary imaging modality used in interventions worldwide. One form of X-ray imaging is angiographic imaging, widely used for vascular interventions (see Figure 1). In theory, the general rendering techniques discussed above are applicable for all vascular visualization. Preim and Oeltze [7] give an overview of 3D vascular visualization. They distinguish between model-based approaches and model-free approaches. Model-based approaches rely on model assumptions to create easy-to-interpret visualizations, while model-free approaches represent the data more faithfully. Ropinski et al. [8] proposed and evaluated several visualization techniques that 


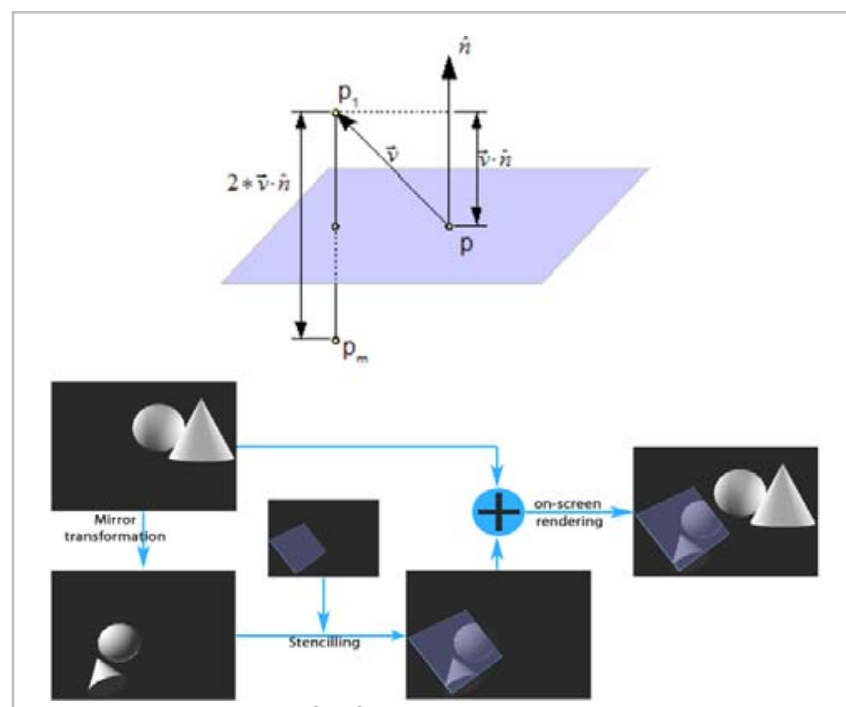

Figure 2: Perception of reflectance. (Top) The mirroring concept and (bottom) stencilling for mirror reflection.

support depth perception of angiogram images. Different depth cues, such as depth-based colour coding, edge enhancement and depth of field effects, are introduced and combined to enhance depth perception of complex vascular systems. Ritter et al. [9] and $\mathrm{Chu}$ et al. [10] proposed a real-time rendering pipeline and efficient modeling for illustrative visualization of vascular system. Still, all of these works are limited to the visual interpretation of 3D data alone.

\subsection{Previous work using virtual mirror}

A first step in achieving simultaneous $2 \mathrm{D}$ and $3 \mathrm{D}$ visualization was the virtual mirror design. This visualization and interaction platform is used only in augmented reality (AR) environments and is specifically crafted for head-mounted displays (HMDs). Its first application was for navigated surgery [11]. Later, the laparoscopic virtual mirror concept was developed as an interaction tool for monitor-based augmented reality [12]. The virtual mirror generates additional mirrored views from any desired perspective on the virtual part of an AR scene [13]. These augmented reality environment systems consist of camera-based tracking and display (i.e. either HMD or monitor). When considering medical data, preoperative CT or MRI can be aligned with the patient and rendered as a virtual part in the $\mathrm{AR}$ environment. The virtual mirror can be attached to a tracked hand-held device, or even to optically tracked instrumentation such as a drilling device or a laparoscope camera [13]. One setback of these settings is that all instruments need to be tracked with added hardware, and line of sight issues need to be considered before effective translation to the operating room.

\subsection{Our contribution}

Although augmented reality in X-ray imaging has been investigated [15-16], no works have conceived the integration of the virtual mirror into the projection geometry of a C-arm fluoroscopy system. Note that X-ray imaging is traditionally an inverse perspective visualization. This fact makes the adaptation innovative and interesting. This work aims at evaluating a new paradigm that encompasses a virtual mirror within the registered 2D/3D overlays during X-ray angiography. We develop a method for interactive visualization of the $3 \mathrm{D}$ data from new perspectives including visualizing a virtual mirror from the same viewpoint as the X-ray source. A feasibility study was designed in the form of a questionnaire to assess the efficacy of a virtual mirror in an X-ray setting. The paper is divided as follows: Section 2 places emphasis on the design, integration and manipulation of the virtual mirror inside an X-ray image, while Section 3 concludes with results and discussion based on the participation of 24 probands that included 10 clinicians.

\section{The Angiographic Virtual MirRor}

This section explains how the virtual mirror is integrated in the $2 \mathrm{D} / 3 \mathrm{D}$ angiographic visualization system. Our initial hypothesis is that virtual mirror can facilitate the localization of aneurysms when visualizing X-ray. Our second hypothesis is that the virtual mirror enhances depth perception when seeing through a region of interest (ROI). Our third hypothesis is that color depth encoding improves the correspondence between $3 \mathrm{D}$ anatomy in mirror and overlay in X-ray. The potential benefit of the virtual mirror in $2 \mathrm{D} / 3 \mathrm{D}$ overlay is providing both static and dynamic views in a single monitor for the interventionalist, without viewing and rotating the $3 \mathrm{D}$ data from a secondary monitor.

\subsection{Perception by reflection}

The most basic issue for mirror realization in computer graphics is to find out the mirror transformation, in other words, to calculate where the objects reflected. In our approach, since the mirror is integrated directly into the $2 \mathrm{D} / 3 \mathrm{D}$ overlay application (directly into the X-ray into the projection geometry), the mirror transformation can be derived from plane-based point mirroring (see Figure 2-top). The derivation of the mirror transformation will be adapted based on [14]. A mirror plane (in 3D) $\pi_{m}$ can be defined by a $3 \mathrm{D}$ point $p$ and normal vector, $\hat{n}$. The problem will then be to find the transformation matrix $M$ using $p$ and $\hat{n}$ such that for the arbitrary $3 \mathrm{D}$ point $p_{I}\left(p_{1} \notin \pi_{m}\right)$, the point $\mathrm{p}_{\mathrm{m}}=M \cdot p_{I}$ is the mirror symmetric point of $p_{l}$ about plane $\pi_{m}$. The derivation follows:

$$
p_{1}-p_{m}=2(\vec{v} \cdot \hat{n}) \times \hat{n}
$$

From Figure $2, \bar{v}=p_{1}-p$, thus we obtain:

$$
p_{m}=p_{1}-2\left(p_{1} \cdot \hat{n}-p \cdot \hat{n}\right) \times \hat{n}
$$

For a fixed plane $\pi_{m}$, the dot product $p \cdot \hat{n}$ is constant. We define $k=p \cdot \hat{n}$, then the relation between $p_{m}$ and $p_{I}$ can be rewritten as:

$$
p_{m}=p_{1}-2 \hat{n}\left(p_{1} \cdot \hat{n}\right)+2 \hat{n} k
$$

By separating the above equation in $\mathrm{x}, \mathrm{y}$ and $\mathrm{z}$ components and distributing by $p_{l x}, p_{l y}$ and $p_{l z}$ we can finally get the mirror transformation matrix $M$ as:

$$
M=\left[\begin{array}{cccc}
1-2 n_{x}^{2} & -2 n_{x} n_{y} & -2 n_{x} n_{z} & 2 n_{x} k \\
-2 n_{y} n_{x} & 1-2 n_{y}^{2} & -2 n_{y} n_{z} & 2 n_{y} k \\
-2 n_{z} n_{x} & -2 n_{z} n_{y} & 1-2 n_{z}^{2} & 2 n_{z} k
\end{array}\right]
$$

In computer graphics, mirror reflection is a standard effect generally realized by two approaches: texture-based mirroring and stencil-buffer mirroring [13]. In this work, the stencil-buffer mirror rendering is used. Stencil buffer is found in modern computer graphics hardware, together with fragment buffer and depth buffer, it is usually used to limit the area of rendering, known as stenciling. The sketch procedure of using stencil buffer for mirroring is shown in Figure 2-bottom. The stencil buffer creates a mask based on the geometry of the mirror. Meanwhile, the mirrored object is rendered offline. During the stenciling, the 

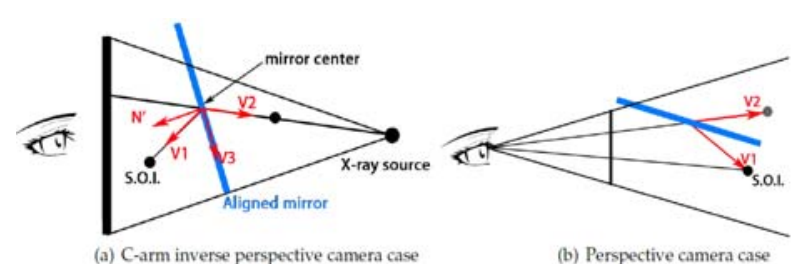

Figure 3: The virtual mirror alignment using C-arm inverse perspective camera and normal perspective camera.

part of the mirrored object that is within the mask, together with the non-mirrored object, is finally rendered onto screen.

\subsection{Virtual Mirror Alignment in X-ray}

Since there is no hand-held device available to manipulate the virtual mirror (which is usually a hard constraint in augmented reality applications [13]), the intuition of the mirror manipulation needs to be enhanced to adapt to the intraoperative setting in angiographic interventions. When using a mirror to interactively observe a 3D structure, the user may prefer to keep the region of interest in the center of the mirror. Interventionalists may manipulate the mirror around some spot of interest (S.O.I.) (e.g. aneurysm, guide-wire or catheter tip) to observe the vessel structure. This requires the automatic alignment of the mirror to the S.O.I. It can be achieved by using two inputs: a viewing matrix as well as the 3D position of the S.O.I. Having the mirrored S.O.I. seen in the center of the mirror means that the mirrored S.O.I. needs to project on the same point as the mirror center does. Figure 3 shows the mirror alignment using two different cameras, the $\mathrm{C}$-arm inverse perspective camera and the perspective camera. The inverse perspective camera is specially used in 2D/3D overlay applications, which simulate the X-ray projective geometry. This scenario is implemented in this work. The X-ray source is the location for the projection. In the inverse perspective case, the mirrored S.O.I. should be mirrored on the line between the mirror center and the X-ray source, but not the line between mirror center and camera position (i.e. interventionalist eye position). Since the mirror center and a normal fix a mirror plane, the mirror alignment is obtained by finding the alignment normal $N^{\prime}$ in Figure $3 \mathrm{a}$. This can be achieved in the following steps:

- $\quad V 3=V 1+V 2$, then normalize $V 3$.

- Rotation axis $V R=\operatorname{cross}(V 1 ; V 2)$, then normalize $V R$.

- $\quad$ New normal $N^{\prime}=\operatorname{cross}(V R ; V 3)$, normalize $N^{\prime}$.

- If the mirror is not facing the viewer, $N^{\prime}=-N^{\prime}$ to flip the mirror.

To check whether the mirror is valid (i.e. facing the viewer), a sign test of the dot product between the viewing direction and the mirror normal vector is sufficient.

\subsection{Perception by occlusion}

Before discussing the interactive manipulation of the virtual mirror, we first describe the steps involved in improving depth perception visualization of vasculature structures in both X-ray and mirror. We begin with the default colour composition in ray casting rendering, which is known as alpha-blending. The main idea behind alpha-blending consists of evaluating the volume rendering integral. The integral is typically along a ray cast from an image pixel and crossing through the volume. The basic integral formula in discrete domain is as follows:

$$
C=\sum_{i=0}^{n} \alpha_{i} C_{i} \prod_{j=0}^{i-1}\left(1-\alpha_{j}\right)
$$

where $\alpha_{i}$ and $C_{i}$ are the opacity and colour values of the $i^{\text {th }}$ sampled voxel $x$. We can also write the formula in the form of a single ith step. The parameters are defined as:

- $\quad$ the colour and the opacity of $i^{\text {th }}$ sampled voxel are $C_{i}$ and $\alpha_{i}$,

- the intermediate colour and opacity before compositing the $i^{\text {th }}$ sample are $\mathbf{C}_{\mathbf{i}-1}$ and an intermediate opacity $\alpha$ value, a.

- the intermediate colour and opacity after compositing the $i^{\text {th }}$ sample are $\mathbf{C}_{\mathbf{i}}$ and $\mathbf{a}_{\mathbf{i}}$.

Then the composition procedure of ith step can be written as:

$$
\begin{aligned}
& C_{i}=C_{i-1}+\left(1-a_{i-1}\right) \cdot C_{i} \cdot \alpha_{i} \\
& a_{i}=a_{i-1}+\left(1-a_{i-1}\right) \cdot \alpha_{i}
\end{aligned}
$$

Equation 6 is known as the standard alpha-blending formula in a single step. Note that the volume is rendered in front-to-back ordering. Our approach of occlusive contour rendering modifies the standard alpha-blending. Three parameters need to be introduced: $\alpha_{i}^{\text {src }}, \alpha_{i}^{\text {occli }}$, and $w$, where $\alpha_{i}^{\text {src }}$ is defined as the opacity value of the ith sample before contour modulation, $\alpha_{i}^{\text {occli }}$ is defined as the occlusive alpha, which is a virtual alpha value used for $\alpha$-composition (where normally the real alpha value is used), and $w$ is in the range $\in[0 ; 1]$ and defined as the occlusion factor controlling the degree of occlusion. Now, the occlusion-adapted alpha-blending is modified as follows:

$$
\begin{aligned}
& C_{i}=C_{i-1}+\left(1-a_{i-1}\right) \cdot C_{i} \cdot \alpha_{i} \\
& a_{i}=a_{i-1}+\left(1-a_{i-1}^{o c c l}\right) \cdot \alpha_{i} \\
& \alpha_{i}^{o c c l}=\alpha_{i-1}^{o c c l}+\left(1-\alpha_{i-1}^{o c c l}\right) \cdot \alpha_{i}^{s r c} \cdot \omega
\end{aligned}
$$

In Equation 7, the occlusive alpha is used for compositing the opacity value. If $w=1$, the following voxel sample in the volume contributes only slightly to the result and the colour will still be transparent. In contrast, if $w \approx 0$, the accumulated occlusive alpha is still near 0 in the next composition. Thus the occlusion factor controls the degree of occlusion.

\subsection{Perception by color depth encoding}

Occlusion is a strong and intuitive depth cue, but works only when overlapping exists between objects. In computer graphics, the $\mathrm{z}$-value of the render element (i.e. vertex) in eye coordinate system is important. It is known as the depth of the render element, and is typically used to generate depth map or for zbuffering. In non-photorealistic rendering (NPR) volume rendering, colour can be modified with featured information. In this section, we describe how the depth value is used for colour coding in order to generate depth perceptive contours. The viewing depth of the voxel is mapped into colour. Depth ratio $\gamma_{d}$ is defined as a parameter linearly associated with depth value:

$$
\gamma_{d}=\frac{D-D_{\text {near }}}{D_{\text {far }}-D_{\text {near }}}
$$

In Equation 8,D is the viewing depth while $D_{\text {near }}$ and $D_{\text {far }}$ are used to define a depth range. Different colour patterns can be used for coding. Previously, pseudo chromadepth technique was used in Ropinski et al. for depth perception in angiography [8]. Here, the 


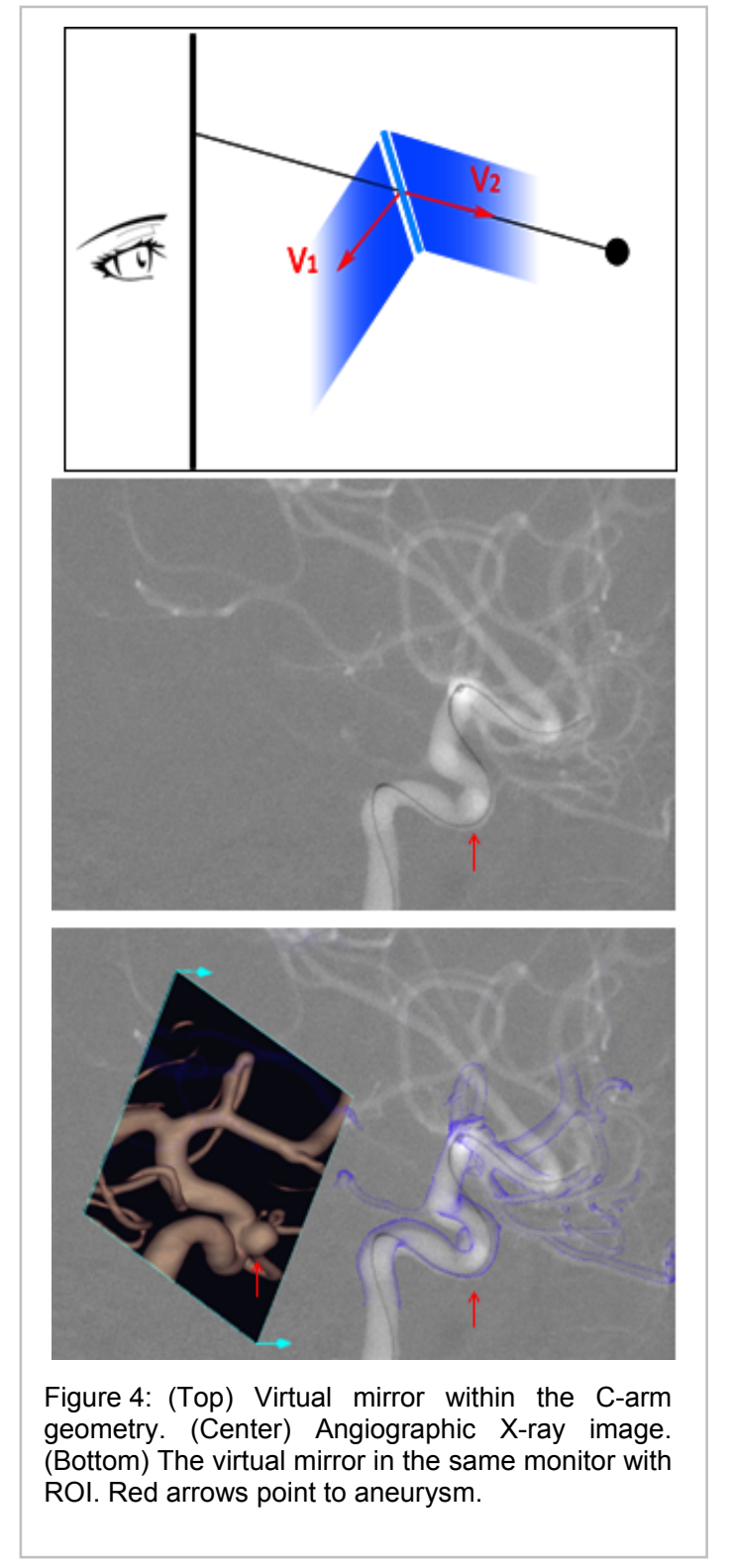

same chromadepth and pseudo chromadepth colour coding are adapted for contour rendering. Given Depth ratio $\gamma_{d} \in[0,1]$ of the $i^{\text {th }}$ sample, the colour $C_{i}$ can be coded based on chromadepth technique as:

$$
C_{i}=\left(1.0-\gamma_{d}, 0.5\left[1-\cos \left(2 \pi \cdot \gamma_{d}\right)\right], \gamma_{d}\right)
$$

Pseudo chromadepth is a reduction of colour hues to only red and blue, the colour $C_{i}$ can be coded as:

$$
C_{i}=\left(1.0-\gamma_{d}, 0, \gamma_{d}\right)
$$

The colour coding defined above uses the RGB colour model. As discussed in [10], too many colour hues may obscure the clarity of the depth perception. Therefore, we investigate the HSI (hue, saturation and intensity) model for colour coding. Both saturation and intensity channels can be coded with $\gamma_{d}$ for depth perception. However, the coded HSI colour needs to be converted to RGB colour for rendering. For good measure, we also investigate an adapting gray value colour that encodes gray value with depth, such as:

$$
C_{i}=\left(1.0-\gamma_{d}, 1.0-\gamma_{d}, 1.0-\gamma_{d}\right)
$$

It is also possible to modulate the opacity value of the contours with $\gamma_{d}$; thus, vasculature farther away becomes more transparent and gives the effect of contours fading as the viewing depth increases. In total, we implement four models for depth encoding: the RGB, RB, HSI, and GRAY colour depth encoding and integrate them to the $2 \mathrm{D}$ anatomy in X-ray and the $3 \mathrm{D}$ anatomy in virtual mirror.

\subsection{Perception by region of interest}

In current clinical practice, an interventionalist can analyze any area within the $3 \mathrm{D}$ vasculature, while clipping out vessels that are not of interest. We define this as R.O.I. The point of interest can be selected by the interventionalist (i.e. such as a bifurcation or an aneurysm) or automatically located (e.g. the position of the tip of the catheter via segmentation and reconstruction algorithms). The size of the R.O.I. is defined by a sphere with a certain diameter. The voxels outside the R.O.I. have a decreased contribution of color and opacity to the final visual result, so the contours outside the R.O.I. will barely be visible to the interventionalist in the final rendered overlay. The R.O.I. of the virtual mirror automatically defines the corresponding region in the $2 \mathrm{D} / 3 \mathrm{D}$ overlay image. As shown in Figure 4-top, the blue plane is the mirror. The users can see the mirrored volume in the center of the mirror along vector $V 2$, which runs from the center of the virtual mirror to the x-ray source. $V 2$ can be transformed using the mirror matrix $\mathrm{T}_{\text {mirror }}$, resulting in the mirrored vector $V 1$. For the overlaid $3 \mathrm{D}$ volume rendering, the depth is in direct proportion to $|z|$ of the voxel in viewing space. Here we define a depth vector as a normalized vector along the depth axis. In the above case, the depth vector is $v \_$depth $=(0,0,-1)$ in viewing space. With the help of the depth vector, we can easily calculate the depth by a dot product, Depth $=$ $\operatorname{dot}(v$ depth, $v$ voxel $)$, where $v$ voxel is the vector from the origin point to the voxel point. In order to have the corresponding color in the mirrored scene, we mirror the depth vector as well as the origin point using the mirror transformation matrix.

\subsection{Virtual Mirror Interactivity}

In section 2.1, the mirror transform matrix was introduced. Here the mirror node based on Open Inventor framework is developed. The mirror node is mainly used to interactively generate the mirror transformation matrix. In Open Inventor, draggers are used to let the interventionalists interact with elements in 3D: scaling, rotating, translation geometry are considered, as well as, lighting. The interaction is done by updating the so-called motion matrix. All draggers take the transformation matrices between the local interactive element (local coordinate system) and the global scene (world coordinate system), which can be obtained using the methods of getLocalToWorldMatrix \& getWorldToLocalMatrix. Our mirror node class (VirtualMirrorDraggerX) makes use of the motion matrix to generate and update the mirror transformation matrix. In the local coordinate system of the mirror element, the point $p_{\text {mirror }}$ and the plane normal $n_{\text {mirror }}$ are selected as the origin of the local coordinate system and $\mathrm{x}$-axis vector, namely: $p_{\text {mirror }}=$ $(0,0,0)$ and $n_{\text {mirror }}=[1,0,0] \mathrm{T}$. By transforming the point $p_{\text {mirror }}$ and vector $n_{\text {mirror }}$ in world coordinate system, the mirror transformation matrix can be easily calculated. The mirror effect is realized using stencil buffer and clip plane. The clip plane can be added to the scene graph. The values of the clip planes are provided by the mirror dragger. It clips out the mirrored part which stretches out of the mirror. Several OpenGL functions that 

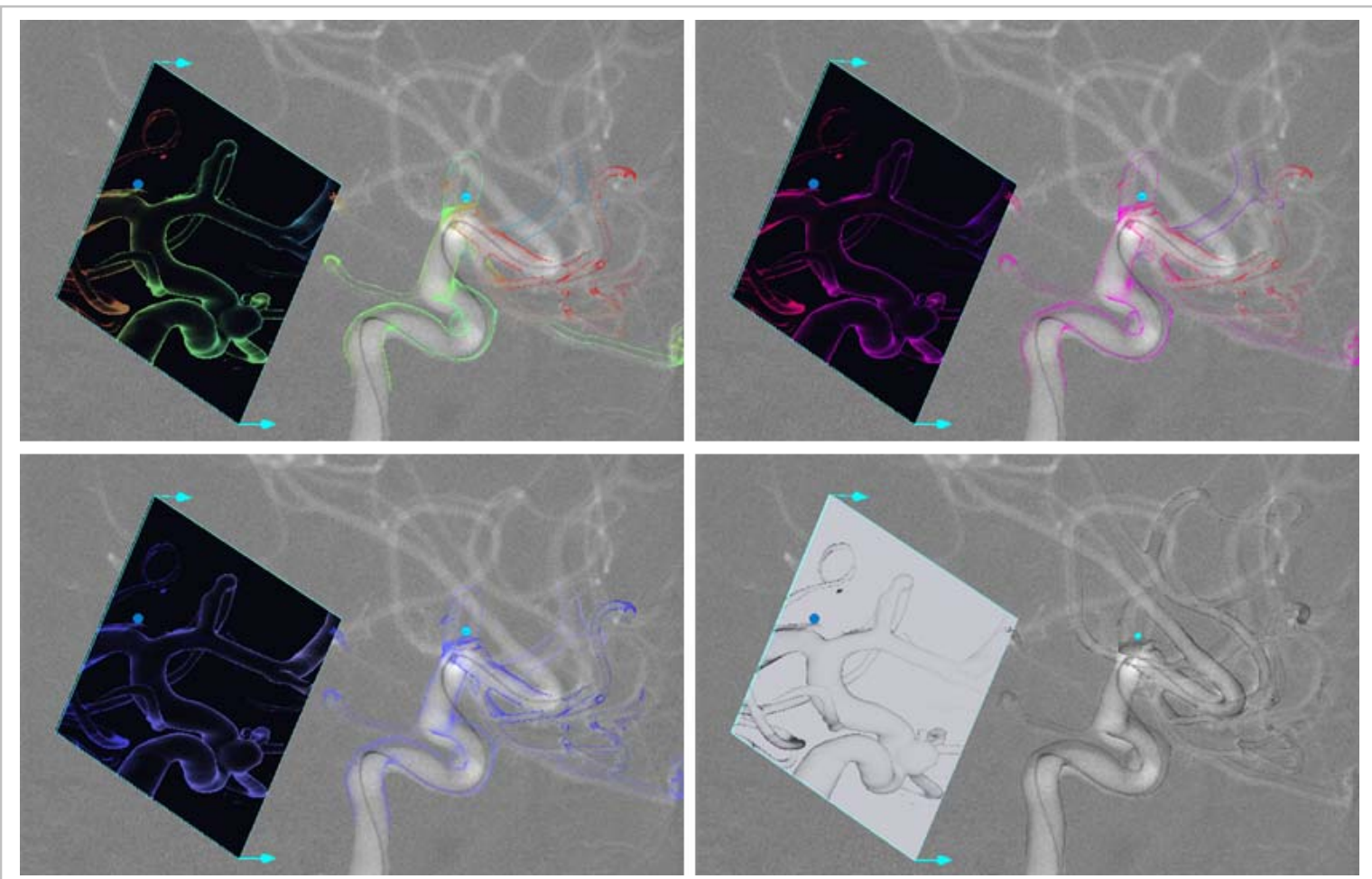

Figure 5: Different color-depth encoding visualization algorithms are implemented to enhance both 2D X-ray and 3D vessels in the virtual mirror. (Top row) These include RGB and RB color-depth encoding as well as (Bottom row) HSI and gray-level depth encoding.

make use of the stencil buffer have been developed for this. Lastly, the mirror is rotated and dragged about a spot of interest as described in Section 2.2. Since this paper is based on a feasibility study, we deem the draggers in OpenInventor sufficient for this initial analysis.

\section{EXPERIMENTS \& RESULTS}

The feasibility of potentially using a virtual mirror in angiographic interventions is evaluated via a survey. A questionnaire is designed where traditional $2 \mathrm{D} / 3 \mathrm{D}$ overlay images, (i.e. those used by surgeons in current clinical practice to navigate surgery), and an overlay including the virtual mirror visualization are evaluated from different aspects. In total, 24 participants were asked to partake: 10 clinicians, 5 medical $R \& D$ engineers and 9 computer scientists.

The survey included questions, ordered randomly by topic, so as to avoid bias. The $2 \mathrm{D} / 3 \mathrm{D}$ overlays, as in Figure 1 , were generated using 1 phantom and 2 clinical datasets. We used Computed tomography angiography (CTA) and rotational angiography for $3 \mathrm{D}$, and the X-ray images for $2 \mathrm{D}$.

A Likert scale is a type of psychometric response scale often used in surveys and is the most widely used scale in survey research. When responding to a Likert questionnaire item, respondents specify their level of agreement to a statement. The scale is named after Rensis Likert (report published in 1932). The format of our 5-pt Likert was: (1) Strongly disagree, (2) Disagree, (3) Neither agree nor disagree, (4) Agree, (5) Strongly agree.

We also considered using a one-way analysis of variance (ANOVA) to compare means of two or more samples (using the $\mathrm{F}$ distribution) [17]. The ANOVA tests the null hypothesis that samples in two or more groups are drawn from the same population. The ANOVA produces the ratio of the variance calculated among the means to the variance within the samples. If the group means are drawn from the same population, the variance between the group means should be lower than the variance of the samples. A higher ratio therefore implies that the samples were drawn from different populations [17]. It also provides significance in the form of a $p$-value (i.e. $<0.05$ implies statistical significance).

\subsection{Can you find the position of an aneurysm?}

Task: We performed a first analysis by asking participants to determine the true position of an aneurysm (i.e. front or back of vessel) shown in 7 different X-ray images, of which 6 are depicted in Figure 6. We repeated the same exercise by including the virtual mirror inside the X-ray image. A red arrow was added in both X-ray and virtual mirror pointing to a potential aneurysm.

Result: Unsurprisingly, only $16.7 \%$ of the participants guessed correctly the position of the aneurysm when looking only at an Xray image. On the other hand, the virtual mirror inside X-ray provided the participants with additional spatial information allowing $100 \%$ of the participants to correctly indicate the position of the aneurysm. The left and right pictures in Figure 6bottom row shows aneurysm positions in back of vasculature whereas all other images depict aneurysm positions in front of vasculature.

\subsection{Does the region of interest help?}

Task: We performed a second analysis by asking participants to determine whether a region of interest (ROI, Section 2.5) enables helps the human eye focus on key areas of the vasculature (i.e. instead of looking at the entire image).

Result: On a five point Likert scale, an average of $3.1 \pm 1.0$ favored the region of interest whereas an average of $2.5 \pm 0.7$ was achieved for excluding. A $p$-value of 0.039 using the ANOVA test shows statistical significance. 


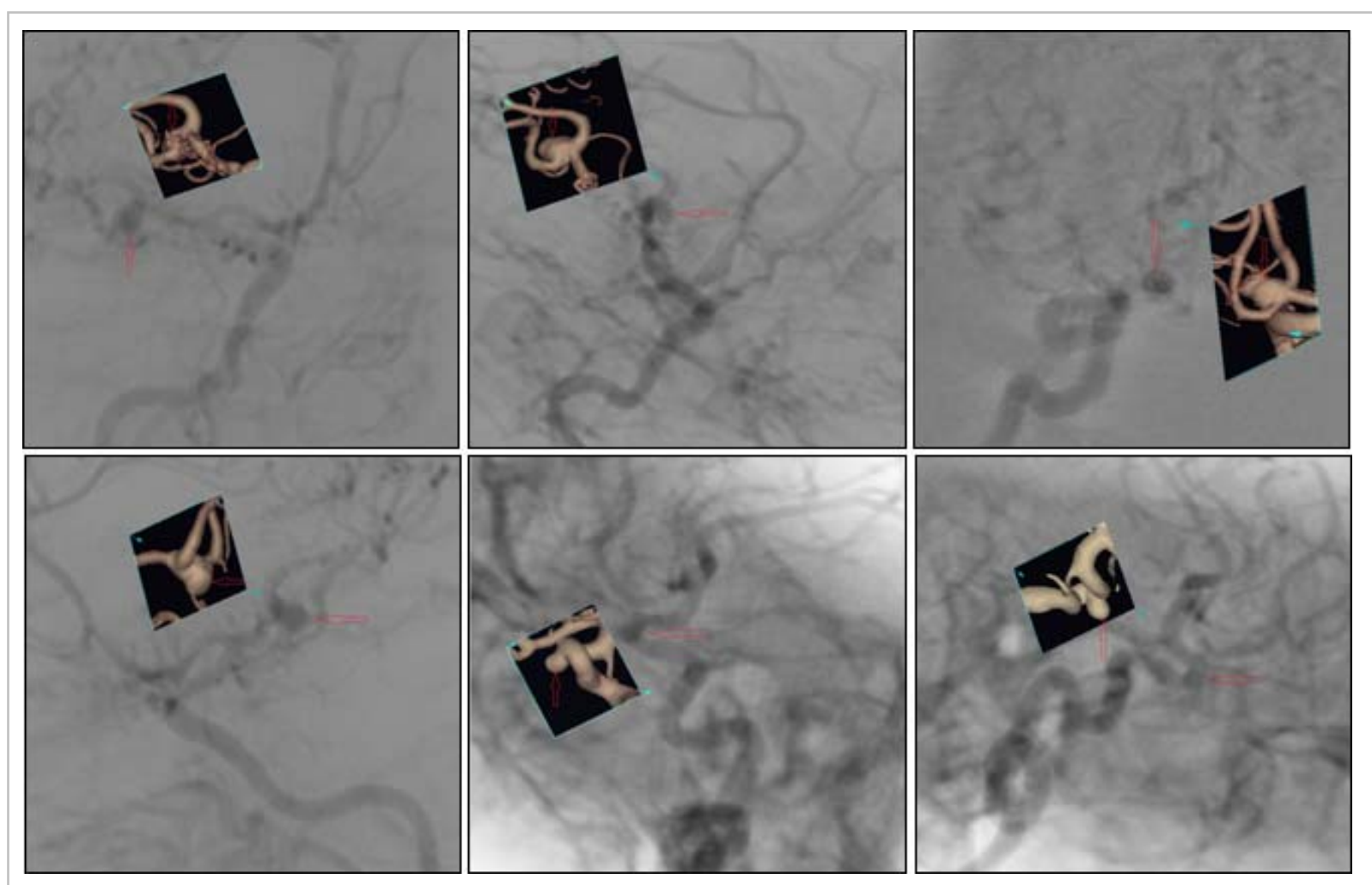

Figure 6: Several examples of user study depicting a virtual mirror inside X-ray image. Participants were asked whether the aneurysm in X-ray is behind or in frontal position of the vasculature. Red arrows are pointing towards the aneurysms in both $X$-ray and virtual mirror. Final results indicate that the virtual mirror made a significant impact in determining the location of the aneurysms.

\subsection{Does color improve vasculature ordering?}

Task: We performed a third analysis on the effect of color depth encoding by asking participants to identify which vessels were farther away or closest to them as depicted in Figure 5. Here, we partitioned the participants into two groups: clinicians and nonclinicians.

Result: Of the 10 clinicians, 7 prefer color-depth encoding (RGB, RB), and 3 prefer GRAY color scheme. On the other hand, all of the non-clinicians prefer a color-coding scheme. Thus, 21 participants agreed or strongly agreed, on a Likert scale, that RGB or RB color-depth encoding helped improve depth perception with respect to vessels being farther or closest to the viewer. Statistical significance from the ANOVA test at $p=0.005$ was measured.

\subsection{Is the virtual mirror intuitive?}

Task: We performed a fourth analysis on how intuitive the virtual mirror is when placed inside the X-ray. Here, the virtual mirror is enhanced with small blue arrows on its rectangular corners, pointing towards the vasculature of interest (see Figure 4bottom)

Result: $70 \%$ of the participating surgeons agreed that the virtual mirror is intuitive inside the X-ray image. However, this positive result was obtained when displaying a GRAY level color encoding such as in Figure 5 bottom row-right. On the other hand, $57 \%$ of non-surgeon participants agreed that the virtual mirror is intuitive. These results were underscored by a non-significant $p$ value after performing the ANOVA test.

\subsection{Can the virtual mirror improve depth perception?}

Task: We performed a fifth analysis on how the virtual mirror augments the original $2 \mathrm{D} / 3 \mathrm{D}$ overlay. We asked the participants to evaluate the virtual mirror in the sense of better understanding 3D structure.

Result: $60 \%$ of the participating surgeons agreed or strongly agreed that the virtual mirror increases depth perception inside the X-ray image. $93 \%$ of non-surgeon participants agreed or strongly agreed that the virtual mirror provides the required depth perception in 3D to compliment the existing 2D X-ray image. A $p$-value of 0.0002 showed statistical significance when performing an ANOVA test.

\subsection{Is virtual mirror interactivity adequate?}

Task: We performed a last analysis to test the feasibility of the virtual mirror. Interactivity was defined as a visual interactive tool in extracting $3 \mathrm{D}$ information from X-ray. In this paper, draggers from OpenInventor were used and filmed in one video sequence. The participants were asked to evaluate the video on how plausible the interactivity looks.

Result: On a five point Likert scale, the participants scored a $3.4 \pm 1.0$ which suggests a positive result on how the virtual mirror is moved inside the X-ray.

\section{Discussion}

Implementing the virtual mirror directly in an X-ray imaging system is the first of its kind. Further, this result is underscored by the fact that the X-ray imaging system is traditionally an inverse 
perspective model. An extension to such an unnatural view of the world is not obvious. We believe that the virtual angiographic mirror concept is not a "simple" extension of the known AR virtual mirror concepts towards a new application and this paper is therefore not simply about its perceptual validation. A feasibility study is proposed in the form of a questionnaire handed to clinician and non-clinician participants. Regarding experiments, new concepts of visualization are often not well accepted by clinicians since they are used to traditional X-ray (grayscale) colors. A good visualization concept should provide a natural understanding of the world. If our concept is accepted by nonclinicians, it means it has potential to ease perceptual training of future surgeons. Clinicians often provide encouraging comments which are not to be taken as granted. The results for the mirror intuition inside the X-ray were not significant. We note that intuitiveness is correlated to learning and experience and therefore such results in the first set of experiments could be expected.

In Section 2, we defined our initial hypothesis as follows: (i) that virtual mirror can facilitate the localization of aneurysms when visualizing X-ray, (ii) the virtual mirror enhances depth perception when seeing a region of interest (ROI), and (iii) color depth encoding improves the correspondence between 3D anatomy in mirror and overlay in X-ray. From our feasibility study, $88 \%$ of total participants agreed that the virtual mirror significantly facilitates the correspondence or association of vessels in $2 \mathrm{D}$ and $3 \mathrm{D}$ simultaneously, in a single monitor.

In clinical practice, the surgeon utilizes a $\mathrm{C}$-arm fluoroscope to guide vascular procedures; therefore, they visualize only X-ray in a monitor. Today, a few commercial packages which offer a 2D/3D overlay to the surgeon (Figure 1) are made available inside the operating room. However, these lack the critical 3D anatomical structures that would help the surgeon treat aneurysms or stenosis in vasculature quickly. The surgeon "mentally" maps the 3D to complete intervention (i.e. based on their many years of training experience). We demonstrated that the virtual mirror absolves this problem as seen in Figure 6.

The current limitations of the virtual mirror, based on our initial feasibility study, include: (i) the inability to visualize sub-surface anatomical structures, (ii) interactivity outside of standard computer mouse has not been implemented yet; (iii) it is difficult to sense the position of the mirror inside the X-ray even with the addition of two arrows on the mirror rectangle.

We also collected the following important comments obtained from some of the participants

- "It can occlude other structures (dependent on the view direction)".

- It is hard to see the position and depth of the mirror in 3D, "which introduces ambiguous".

- And it is also "difficult to relate the scene shown on the mirror to their displayed $3 D$ structures without a ROI".

- The virtual mirror is helpful: to "see hidden structures".

- The virtual mirror is useful for "complex aneurysms and structures such as in the human head".

\section{FUTURE WORK ON IMPROVING THE VIRTUAL MIRROR}

Future work will focus on developing an improved shape configuration of the mirror such as a circular configuration, or a rectangular shape with an enlarged thickness, allowing humans to interpret 3D location of the mirror with increased intuition. We will also consider using a wireless mouse control to maneuver the mirror inside X-ray [13]; other alternatives proposed in literature might include using Kinect or inertial sensors. In this first feasibility study, the results indicate that the virtual mirror has the potential to play a great role in angiography rooms. Although, color depth encoding received positive feedback from the participants, 3 surgeons still relied on a GRAY color depth encoding to answer the questions; this suggesting that surgeons would have to adapt and move away from the conventional gray scale X-ray visualizations found in current clinical practice. Interestingly, none of the 10 surgeons were discouraged by this allowing our technique to be a hopeful alternative in the operating room in the near future. Lastly, we are currently visualizing only preoperative 3D anatomy, from MRI or CT, inside the mirror. This is a crucial limitation as the surgeon will not perceive a guidewire or catheter in real-time as is the case to them when looking at the intraoperative monitors from a $\mathrm{C}$-arm fluoroscope. To remedy this, we propose to investigate the use of a virtual mirror intraoperatively using either monoplane or biplane $\mathrm{C}$-arm fluoroscopes. Although angiographic interventions is the primary targeted clinical application, the virtual mirror can be integrated to other C-arm fluoroscopy based procedures such as in cardiac ablation for the treatment of arrhythmias [18-20].

\section{Conclusion}

Due to the virtual mirror visualization device being positioned inside the same monitor in angiographic applications, the 3D part of the visualization can be intuitively perceived and additional information about the $3 \mathrm{D}$ structure can be explored interactively by the interventionalist. The virtual mirror can be positioned into the scene and fits into the viewing projective geometry of the angiography imaging system. A user interface allows the user to move the virtual mirror which in turn reflects the registered angiographic data. This allows the visualization of the complex $3 \mathrm{D}$ anatomical structures including $3 \mathrm{D}$ vessels without disturbing the alignment of $2 \mathrm{D} X$-ray with the $3 \mathrm{D}$ data. The $3 \mathrm{D}$ data can be obtained from CT angiography, MRI, or any other source of imaging. This novel concept for X-ray angiographic visualization has the potential to impact the angiographic room of the future.

\section{REFERENCES}

[1] Markelj, P., Tomazevic, D., Likar, B., Pernus, F.: A review of 3d/2d registration methods for image-guided interventions. Medical Image Analysis (2010)

[2] S. Bruckner and ME Groller. Enhancing depth-perception with flexible volumetric halos. Visualization and Computer Graphics, IEEE Transactions on, 13(6): pp.1344-1351, (2007)

[3] B. Mora and D.S. Evert. Instant volumetric understanding with orderindependent volume rendering. In Computer Graphics Forum, volume 23, pp. 489-497. Wiley Online Library, (2004)

[4] M.A. Kersten, A.J. Stewart, N. Troje, and R. Ellis. Enhancing depth perception in translucent volumes. Visualization and Computer Graphics, IEEE Transactions on Visualization and Computer Graphics, 12(5): pp.1117-1124, (2006)

[5] M. Wieczorek, A. Aichert, P. Fallavollita, O. Kutter, A. Ahmadi, L. Wang, and N. Navab. Interactive $3 \mathrm{~d}$ visualization of a single-view $\mathrm{X}$ ray image. Medical Image Computing and Computer-Assisted Intervention-MICCAI 2011, pp. 73-80, (2011)

[6] C. Boucheny, G.P. Bonneau, J. Droulez, G. Thibault, and S. Ploix. A perceptive evaluation of volume rendering techniques. ACM Transactions on Applied Perception (TAP), 5(4): pp.23, (2009)

[7] B. Preim and S. Oeltze. 3d visualization of vasculature: an overview. Visualization in medicine and life sciences, pp 39-59, (2008)

[8] T. Ropinski, F. Steinicke, and K. Hinrichs. Visually supporting depth perception in angiography imaging. In Smart Graphics, pp. 93-104. Springer, (2006)

[9] F. Ritter, C. Hansen, V. Dicken, O. Konrad, B. Preim, and H.O. Peitgen. Realtime illustration of vascular structures. Visualization and Computer Graphics, IEEE Transactions on, 12(5): pp.877-884, (2006)

[10] A. Chu, W.Y. Chan, J. Guo, W.M. Pang, and P.A. Heng. Perceptionaware depth cueing for illustrative vascular visualization. 
BioMedical Engineering and Informatics, International Conference on, volume 1, pp. 341-346 (2008)

[11] C. Bichlmeier, T. Sielhorst, and N. Navab. The tangible virtual mirror: New visualization paradigm for navigated surgery. In AMIARCS - The Tangible Virtual Mirror: New Visualization Paradigm for Navigated Surgery, Copenhagen, Denmark, MICCAI Society. (2006)

[12] N. Navab, M. Feuerstein, and C. Bichlmeier. Laparoscopic virtual mirror - new interaction paradigm for monitor based augmented reality. In Virtual Reality, pp. 43-50, Charlotte, North Carolina, USA, March (2007)

[13] C. Bichlmeier, S.M. Heining, M. Feuerstein, and N. Navab. The virtual mirror: a new interaction paradigm for augmented reality environments. Medical Imaging, IEEE Transactions on, 28(9): pp. 1498-1510, (2009)

[14] K. Samyn. Mirroring a point on a 3d plane. http://knol.google.com/ k/mirroring-a-point-on-a-3d-plane. [Online accessed March 1,2012]

[15] Masutani Y, Dohi T, Yamane F, Iseki H, Takakura K. Augmented reality visualization system for intravascular neurosurgery. Comput Aided Surg 3(5): pp. 239-47, (1998)

[16] Chen X, Wang L, Fallavollita P, Navab N. Precise X-ray and video overlay for augmented reality fluoroscopy. Int $\mathrm{J}$ Comput Assist Radiol Surg. May 17, (2012) [Epub ahead of print]

[17] Howell, David. Statistical Methods for Psychology. Duxbury. pp. 324-325. ISBN 0-534-37770-X (2002).

[18] P. Fallavollita. Acquiring Multiview C-Arm Images to Assist Cardiac Ablation Procedures. European Association for Signal Processing: Journal on Image and Video Processing, vol. 2010, Article ID 871409, 10 pages, doi:10.1155/2010/871409, (2010)

[19] P. Fallavollita. Is Single-View Fluoroscopy Sufficient in Guiding Cardiac Ablation Procedures? International Journal of Biomedical Imaging, vol. Article ID 631264, 13 pages, (2010)

[20] P. Fallavollita. 2D/3D Registration of Mapping Catheter Images for Arrhythmia Interventional Assistance. IJCSI International Journal of Computer Science Issues, Vol. 4, No. 2, pp.10-19, (2009) 